\section{Aspectos legais do aleitamento materno: cumprimento da lei por hospitais de médio e de grande porte de Maceió}

\section{Legal aspects of breast-feeding: law compliance in medium and large hospitals in Maceió}

Roseane Leite de Oliveira 1

Adriana Nobre Silva 2

1 Programa de Saúde da Família. Conjunto Penedo, 35, Feitosa, Maceió, AL, Brasil. CEP: 57.043-350. E-mail:

roseane_enfermeira@hotmail.com

2 Programa de Saúde da Família. Joaquim Gomes, Alagoas.

\begin{abstract}
Objectives: to assess compliance to Brazilian legislation regarding breast-feeding in medium and large hospitals in the municipality of Maceió in 2000.

Methods: descriptive study selecting large and medium sized, public and private hospitals in Maceio employing at least 30 women over 16 years old. The following variables were established: number of employees, availability of breast-feeding facilities and of cribs in each of the hospitals. Data were collected through semi-structured interviews based on current legislation, and visitation of locations where children being nursed were kept.

Results: a) 17 hospitals grant the 120 days maternity leave and the two 30 minutes break for breastfeeding purposes according to legislation; b) 10 do not have any facilities for the babies or their employees (mothers); c) as for day care centers none follow the basic rules established by law.

Conclusions: hospitals are following only part of the law and there is no interest in the part of authorities in charge of enforcing the laws assuring breastfeeding.

Key words Breast feeding, Legislation, Brazil
\end{abstract}

\section{Resumo}

Objetivos: verificar o cumprimento da legislação brasileira sobre o aleitamento materno por hospitais de médio e grande porte no município de Maceió, durante o ano 2000.

Métodos: estudo descritivo para o qual foram selecionados os hospitais públicos e privados de médio e grande porte de Maceió em que trabalhassem pelo menos 30 mulheres com mais de 16 anos de idade. As seguintes variáveis foram determinadas: número de funcionárias, disponibilidade de locais de amamentação e de berços em cada hospital. Os dados foram colhidos através de entrevista semi-estruturada, baseada na legislação vigente, e em visitas aos locais de guarda dos filhos das nutrizes.

Resultados: a) 17 hospitais concedem os 120 dias de licença-maternidade e os dois descansos de 30 minutos cada, destinados à amamentação conforme legislação; b) 10 não têm local reservado para os bebês de suas funcionárias; c) em relação as creches, nenhuma segue as normas básicas de funcionamento estabelecidas na legislação.

Conclusões: os hospitais cumprem só parcialmente a legislação e existe descaso dos órgãos competentes na fiscalização do cumprimento das leis de proteção ao aleitamento materno.

Palavras-chave Aleitamento materno, Legislação, Brasil 


\section{Introdução}

A importância do aleitamento materno exclusivo até os seis meses é um fato amplamente reconhecido. A Organização Mundial da Saúde e as instituições e órgãos não-governamentais que estudam e divulgam este assunto são unânimes em sua orientação: a amamentação é essencial no primeiro ano de vida, mas, de preferência, deve continuar até a criança completar pelo menos dois anos.

Mesmo sendo o leite o alimento mais indicado para as crianças, principalmente em seus primeiros seis meses de vida, vários estudos têm mostrado, em algumas situações, uma redução no tempo de amamentação exclusiva, que se deve a vários fatores, como por exemplo a falta de preparação das mulheres para a amamentação durante o pré-natal. ${ }^{1}$ Além disso, acredita-se que, nas áreas urbanas, uma das razões do desmame precoce é a separação da mãe de seu filho, devido à volta da mulher ao trabalho fora do lar, condição esta agravada nas grandes cidades pelas precárias condições de transporte e a distância entre a residência e o local de trabalho. ${ }^{2}$

O papel da mulher dentro da sociedade capitalista, sendo obrigada a trabalhar fora de casa contribui grandemente para o abandono da amamentação. Este problema é agravado pelos altos índices de desemprego, que geram uma necessidade cada vez maior de contribuição financeira da mulher na sustentação da família. ${ }^{1}$

A prática do aleitamento materno, além de poupar gastos com a saúde de bebês por diminuir consideravelmente o aparecimento de patologias infantis, proporciona benefícios econômicos para as empresas, reduzindo ausências de mães trabalhadoras.

Várias pesquisas revelam que os empregadores que respeitam as leis trabalhistas e facilitam a amamentação no local de trabalho, contam com funcionárias mais interessadas e com menores índices de falta, porque as mães não necessitam se preocupar com bebês doentes, aumentando assim a sua produtividade. Nos Estados Unidos, programas de empresas que apoiam a amamentação reduziram em $27 \%$ as faltas ao trabalho e em $36 \%$ os custos de cuidados com a saúde. ${ }^{3}$ No México, um projeto de promoção do aleitamento materno num hospital custa apenas quatro dólares por bebê, o que representa muito menor gasto do que intervenções como reidratação oral e vacinação. 3

Pelo exposto, e sendo os hospitais instituições que empregam, na maioria, profissionais de saúde, o presente trabalho pretende identificar o cumprimento das leis asseguradas pela Constituição Brasileira e Consolidação das Leis Trabalhistas (CLT) para a proteção aos direitos da nutriz funcionária de hospitais de médio e grande porte.

\section{Métodos}

Trata-se de um estudo descritivo que avaliou o cumprimento da legislação brasileira quanto ao aleitamento materno por funcionárias de hospitais da cidade de Maceió, AL, Brasil.

A partir da lista de hospitais de médio porte (50 a 149 leitos) e grande porte (150 a 490 leitos) da cidade, fornecida pelo Sindicato dos Hospitais do município foram os mesmos visitados, informados sobre a pesquisa e consultados sobre seu interesse em participarem do estudo. Em seguida, averiguouse o número de mulheres entre 16 e 40 anos que neles trabalhavam, desde que, conforme a CLT (1997; artigo 389, título III, capítulo III, seção IV, parágrafo $1^{\text {o: }}$ 255): "... os estabelecimentos em que trabalhem pelo menos trinta mulheres com mais de dezesseis anos de idade terão local apropriado onde seja permitido às empregadas guardar sob vigilância e assistência os seus filhos no periodo da amamentação." 4

Dos 20 hospitais de médio e grande porte identificados, 17 foram escolhidos para a pesquisa, visto que dois deles não se enquadravam no citado decreto-lei, e um recusou-se a participar da pesquisa.

Os 17 hospitais foram novamente visitados para preenchimento - junto ao setor de recursos humanos ou à coordenação de enfermagem - de um formulário com perguntas a respeito do cumprimento de normas ditadas por lei que asseguram às mães trabalhadoras o direito de amamentar seus filhos usufruindo de licença-maternidade de 120 dias, dois descansos de trinta minutos em cada turno de trabalho e de creche (que pode ser própria ou conveniada, mas próxima ao local de trabalho).

Nos hospitais que possuíam ou mantinham convênio com uma creche, foram ainda entrevistadas três funcionárias de diferentes funções que utilizavam os serviços de creche, para verificar a ratificação das informações fornecidas pelos respectivos hospitais; foi garantido o anonimato tanto dos estabelecimentos quanto das funcionárias. Foram também visitadas as creches referidas pelas funcionárias, para observação e conhecimento do local quanto ao cumprimento da legislação.

A distribuição de frequiência de hospitais públicos e privados, da disponibilidade de local próprio para amamentação, de creches, de funcionárias entre 16 e 40 anos e de berços disponíveis foi determinada. Os dados foram descritos como freqüências absolutas e relativas. 


\section{Resultados}

Na classificação por categoria, observa-se que 11 (65\%) dos 17 hospitais são da rede privada enquanto seis (35\%) são da rede pública. Quanto ao porte, 12 (71\%) são de médio e cinco (29\%) grande (Tabela 1).

O direito aos 120 dias de licença-maternidade, é acatado pela totalidade dos hospitais, os quais permitem a flexibilização nas negociações dos descansos especiais de forma que possam melhor atender às necessidades de mãe-filho.

Conforme indicado na Tabela 2, 10 (59\%) hospitais não oferecem às funcionárias um local apropriado para a guarda de seus filhos durante o período mínimo de seis meses para amamentação, conforme estabelece a lei.

De acordo com a Tabela 3 verifica-se que dos sete hospitais que proporcionam local adequado para guar- da dos filhos das funcionárias, cinco mantêm convênio com creches próximas, um tem creche em sua sede e um tem creche própria instalada em outro local. No que se refere à distância estabelecida entre as instituições e as creches, cinco (72\%) das creches estão localizadas a uma distância inferior a 100 metros da instituição, uma (14\%) de 100 a 200 metros e uma (14\%) está a uma distância superior a 200 metros. Nenhum dos hospitais cobra qualquer taxa da funcionária pela prestação do serviço de creche ou pela manutenção do funcionamento da mesma. Quanto à idade permitida para permanência da criança na creche, observou-se que em uma é de até seis meses de idade e nas demais ultrapassa os seis meses. Porém nenhuma das creches possui os requisitos básicos para o funcionamento legal, isto é, pelo menos uma saleta de amamentação além de um número adequado de leitos no berçário (Tabela 4).

Tabela 1

Classificação dos hospitais estudados. Maceió, AL, Brasil.

\begin{tabular}{|c|c|c|c|c|c|c|}
\hline Hospitais & $\mathrm{n}$ & $\%$ & $\mathrm{n}$ & $\%$ & $\mathrm{n}$ & $\%$ \\
\hline Médio porte (50 a 149 leitos) & 4 & 33,0 & 8 & 67,0 & 12 & 71,0 \\
\hline Grande porte (150 a 490 leitos) & 2 & 40,0 & 3 & 60,0 & 5 & 29,0 \\
\hline
\end{tabular}

Tabela 2

Hospitais que garantem um local apropriado para guarda dos filhos das funcionárias durante o período mínimo de amamentação. Maceió, AL, Brasil, 2000.

\begin{tabular}{lrr} 
Garantia do local & $\mathbf{n}$ & $\%$ \\
\hline Não garantem & 10 & 59,0 \\
Garantem & 7 & 41,0 \\
Total & 17 & 100,0 \\
\hline
\end{tabular}


Número de creches conforme as opções adotadas pelos hospitais que garantem local adequado para guarda dos filhos das funcionárias. Maceió, AL, Brasil, 2000.

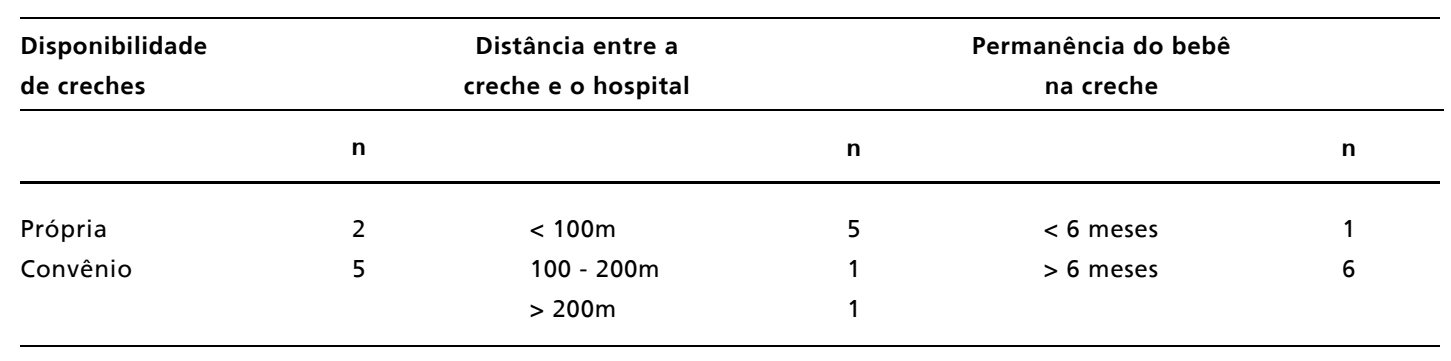

\section{Tabela 4}

Número de leitos no berçário da creche x número de funcionárias da instituição com idades entre 16 a 40 anos. Maceió, AL, Brasil, 2000.

\begin{tabular}{|c|c|c|c|c|c|}
\hline \multirow{2}{*}{$\begin{array}{l}\text { Hospitais que } \\
\text { possuem / mantêm } \\
\text { convênio com } \\
\text { creches }\end{array}$} & \multirow{2}{*}{$\begin{array}{c}\text { Funcionárias } \\
\text { (16 a } 40 \text { anos) } \\
n\end{array}$} & \multirow{2}{*}{$\begin{array}{c}\begin{array}{c}\text { Berços } \\
\text { obrigatórios } \\
\text { por lei }\end{array} \\
n\end{array}$} & \multirow{2}{*}{$\begin{array}{c}\text { Berços } \\
\text { disponíveis na } \\
\text { creche }\end{array}$} & \multicolumn{2}{|c|}{$\begin{array}{c}\text { Berços } \\
\text { disponíveis / Berços } \\
\text { obrigatórios }\end{array}$} \\
\hline & & & & $\mathrm{n} / \mathrm{m}$ & $\%$ \\
\hline Hospital 1 & 62 & 2 & 2 & $2 / 2$ & 100,0 \\
\hline Hospital 2 & 102 & 3 & 2 & $2 / 3$ & 67,0 \\
\hline Hospital 3 & 153 & 5 & 3 & $3 / 5$ & 60,0 \\
\hline Hospital 4 & 180 & 6 & 6 & $6 / 6$ & 100,0 \\
\hline Hospital 5 & 213 & 7 & 6 & $6 / 7$ & 87,0 \\
\hline Hospital 6 & 260 & 9 & 6 & $6 / 9$ & 67,0 \\
\hline Hospital 7 & 825 & 28 & 11 & $11 / 28$ & 40,0 \\
\hline
\end{tabular}

\section{Discussão}

Mesmo existindo uma legislação que dá direito às mulheres trabalhadoras de amamentar seu filho por pelo menos até o sexto mês de vida, esse benefício não é atendido o suficiente para que o aleitamento materno seja um verdadeiro sucesso. Todos os hospitais de médio e grande porte incluídos neste estudo cumprem apenas parcialmente as leis que dão proteção ao trabalho da mulher e garantem seu direito à maternidade.

O cumprimento é rigoroso apenas no que se refere às disposições das leis contidas na Constituição da República Federativa do Brasil (1988: título II, capí- tulo II, artigo $7^{\circ}$, inciso XVIII) $)^{5}$ "... licença à gestante, sem prejuízo do emprego e do salário, com a duração de cento e vinte dias ..." e da Consolidação das Leis Trabalhistas, (CLT: título III, capítulo III, seção V, artigo 396)6 "Para amamentar o próprio filho, até que este complete 6 (seis) meses de idade, a mulher terá direito, durante a jornada de trabalho, a 2 (dois) descansos especiais, de meia hora cada um ...".

Mas é preocupante constatar que a maioria das instituições hospitalares não asseguram às mães trabalhadoras a amamentação de seus filhos durante o período estabelecido em lei, por não lhes oferecer um local apropriado para guarda dos lactentes (CLT: 
título III, capítulo III, seção IV, artigo $\left.389,1^{\circ} \S\right)^{6}$ "Os estabelecimentos em que trabalhem pelo menos 30 (trinta) mulheres com mais de 16 (dezesseis) anos de idade terão local apropriado onde seja permitido às empregadas guardar sob vigilância e assistência os seus filhos no periodo de amamentação ...", o que dificulta para essas mulheres a sua saída da instituição e ir até em casa para amamentá-los. Diante disso, esses hospitais estabelecem com a funcionária a flexibilização de horários como uma estratégia para manter o prolongamento do aleitamento materno, e proporcionam à lactante as seguintes opções: 1) chegar trinta minutos após o início de cada turno; 2) sair trinta minutos antes do término de cada turno; 3 ) chegar uma hora após o início de um dos turnos, ou 4) sair uma hora antes do término de um dos turnos. Em estudo sobre aleitamento materno na Universidade Estadual de Campinas, Hardy et al. ${ }^{7}$ observaram que esses prazos são insuficientes e motivam as servidoras a fazerem concessão de horários especiais nos períodos de amamentação visto que incluem também o tempo que a mãe gasta para ir à creche e chegar ao seu local trabalho. Rea et al. ${ }^{2}$ documentaram, em pesquisa com trabalhadoras em indústrias de São Paulo, que mulheres da área metropolitana, por não conseguirem sair da empresa e ir para casa amamentar, preferem negociar as pausas podendo até conseguir não trabalhar nos fins de semana enquanto o bebê é pequeno, aproveitando para amamentar em livre demanda.

As creches nos locais de trabalho permitem a proximidade entre a mãe e o filho e tendem a manter a amamentação por um período de tempo mais prolongado, embora este estudo tenha encontrado apenas uma creche com estas características. Morse et $a l .{ }^{8}$ através de um estudo com mulheres de diferentes ocupações remuneradas no Canadá, relataram que a possibilidade da mãe ter seu bebê por perto, e acesso a ele no decorrer do período de amamentação, foram os fatores que mais contribuíram para o sucesso do aleitamento. Isto, porém, não foi verificado na presente pesquisa.

Ainda no artigo 389 da CLT, 6 o $2^{\circ} \S$ se diz que "... a exigência do parágrafo $1^{\circ}$, poderá ser suprida por meio de creches distritais mantidas, diretamente ou mediante convênios, com outras entidades públicas ou privadas, pelas próprias empresas, em regime comunitário, ou a cargo do SESI, do SESC, da LBA ou de entidades sindicais", o que ficou constatado na maioria dos estabelecimentos hospitalares desta pesquisa em que não havia creche na própria instituição.

Quanto às creches situadas fora do local de trabalho, seis delas estavam localizadas a uma distân- cia máxima de 200 metros da instituição, não sendo assim um fator que dificultasse a ida da mãe à creche, além de proporcionar a manutenção da amamentação. Rea et al.,2 relatam que, quando comparado o tempo de duração da amamentação entre as trabalhadoras sem creche e com creche no local de trabalho, não foi encontrada nenhuma influência neste aspecto.

No entanto, visto que as empresas não fornecem transporte para o deslocamento da funcionária até a creche, é possível que isso resulte na redução do tempo destinado ao aleitamento.

Entre os hospitais que optaram por creches conveniadas, apenas uma das creches está localizada a uma distância superior a 200 metros, tornando-se inútil para o objetivo a que se propõe.

Constatou-se ainda que nenhuma das creches oferecidas pelos hospitais cumpria integralmente as normas mínimas legais para o funcionamento de qualidade, ou seja, identificou-se a inexistência de saleta de amamentação e, em vários hospitais o número de leitos no berçário da creche é insuficiente com relação ao número de funcionárias do hospital, contrapondo-se assim às disposições encontradas na CLT (título III, capítulo III, seção V, artigo 400):6 "Os locais d estinados à guarda dos filhos das operárias durante o período de amamentação deverão possuir, no mínimo, um berçário, uma saleta de amamentação, uma cozinha dietética e uma instalação sanitária”, e da portaria número um da Divisão Nacional de Saúde e Higiene do Trabalho (DNSHT) de 1969, que aponta dentre outros itens, que o número de leitos no berçário deve obedecer a uma proporção de um para cada grupo de trinta empregadas de 16 a 40 anos de idade. ${ }^{7}$

Diante dos resultados desse estudo, constatamos ainda que a realidade na Região aqui investigada não mudou quando o comparamos ao realizado em âmbito nacional, em 1981, e citado por Rego, 9 no qual se verificou apenas $2 \%$ de cumprimento dessas leis e o "... restante ia desde a burla até o pagamento de multas sempre inferiores ao custo de uma creche."

Por outro lado, o estado de Santa Catarina conseguiu um índice em torno de $85 \%$ no cumprimento dessas leis, fazendo cumprir a exigência de registro de creches próprias e conveniadas na Delegacia Regional do Trabalho: obrigatoriedade da empresa em proporcionar às mulheres o conhecimento de seus direitos e o modo como utilizá-los, bem como modificação do tipo de inspeção e controle por visitas às creches e empresas, além da divulgação desses direitos pelos meios de comunicação e de campanhas educativas a fim de aumentar o cumprimento das leis existentes. 


\section{Referências}

1. Bezerra AEM. Desmame precoce: fatores sociais que o provocam Maceió: Universidade Federal de Alagoas; 1993.

2. Rea, MF, Venâncio SI, Batista LE, Santos RG, Greiner T. Possibilidades e limitações da amamentação entre mulheres trabalhadoras formais. Rev Saúde Pública 1997; 31: 149-56.

3. WABA (World Alliance for Breastfeeding Action ). Amamentar é um barato. Disponível em: 〈http: www.waba.org.br〉. Acesso em: [2000 jan 18].

4. Saad, EG. Consolidação das Leis do Trabalho: comentada. 30. ed. atualizada. São Paulo: LTr; 1997.

5. Brasil. Constituição, 1988. Disponível em: <http://www.geocities.com/paginajuridica /index.html>. Acesso em: [2003 fev 8].
6. CLT (Consolidação das Leis do Trabalho). Disponível em: <http://www.crystalnet.com.br/ codigos/g0000010.html>. Acesso em: [2003 fev 8].

7. Hardy EE, Sandoval LEM, Pinotti JA. Aleitamento materno: um direito da mãe e da criança. Rev Paul Medicina 1987; 105: 103-7.

8. Morse JM, Bottorff JL, Boman J. Patterns of breastfeeding and work: the Canadá experience. Can J Public Health 1987; 80: 182-7.

9. Rego JD. Proteção ao aleitamento materno: o papel das leis ou as leis do papel? J Pediatr [Rio de Janeiro] 1983; 55: 329.

Recebido em 26 de maio de 2001

Versão final reapresentada em 6 de setembro de 2002 Aprovada em 7 de novembro de 2002 